\title{
ON PRODUCTS OF SUMMABILITY METHODS
}

\section{OTTO SZÁSZ}

1. At the recent International Congress I. M. Sheffer asked me the following question: Given a sequence $\left\{s_{n}\right\}$; form the sequence $\left\{\sigma_{n}^{\alpha}\right\}$ of the Cesàro means of order $\alpha(\alpha>0)$ corresponding to $\left\{s_{n}\right\}$. If $\left\{s_{n}\right\}$ is summable Abel, is it true that $\left\{\sigma_{n}^{\alpha}\right\}$ is also summable Abel?

A more general question is: Suppose that $A$ and $B$ are two regular summability methods for sequences $\left\{s_{n}\right\}$. Denote by $A B$ the iteration product which associates with a given sequence the $A$ transform of its $B$ transform; when does $A$ summability imply $A B$ summability?

We shall show in $\S 2$ that the answer is affirmative when $B$ is $(C, \alpha)$ and $A$ is Abel summability. ${ }^{1}$ In $\S 3$ we generalize this result to Laplace transforms and Riesz summability. In $\$ 4$ we discuss the iteration product of Cesàro and Borel summability, and also Euler and Borel summability.

2. Let $f(x)=\sum_{0}^{\infty} a_{n} x^{n}=(1-x) \sum s_{n} x^{n}$. Abel summability of $\sum a_{n}$ to $s$ is

$$
A \lim s_{n}=\lim _{x \rightarrow 1}(1-x) \sum s_{n} x^{n}=s .
$$

We define $s_{n}^{\alpha}$ by the identity

$$
f(x)=(1-x)^{\alpha+1} \sum_{0}^{\infty} s_{n}^{\alpha} x^{n},
$$

and $\gamma_{n}^{\alpha}$ by

$$
(1-x)^{-\alpha-1}=\sum_{0}^{\infty} \gamma_{n}^{\alpha} x^{n}
$$

so that

$$
\gamma_{n}^{\alpha}=C_{n+\alpha, n}=\frac{\Gamma(\alpha+n+1)}{n ! \Gamma(\alpha+1)}
$$

We now have

$$
\sigma_{n}^{\alpha}=\frac{s_{n}^{\alpha}}{\gamma_{n}^{\alpha}}, \quad s_{n}^{\alpha}=\sum_{v=0}^{n} s_{v} \gamma_{n-v}^{\alpha-1}
$$

Presented to the Society, April 27, 1951; received by the editors February 7, 1951.

${ }^{1}$ For the case $\alpha=1$ see [4, p. 189]; [3, p. 258]. Numbers in brackets refer to the literature at the end of this paper. 
Furthermore

$$
\frac{1}{\gamma_{n}^{\alpha}}=\alpha \int_{0}^{1} \rho^{n}(1-\rho)^{\alpha-1} d \rho
$$

hence

$$
\begin{aligned}
\sum_{0}^{\infty} \sigma_{n}^{\alpha} x^{n} & =\alpha \int_{0}^{1}(1-\rho)^{\alpha-1} \sum s_{n}^{\alpha}(\rho x)^{n} d \rho \\
& =\alpha \int_{0}^{1}(1-\rho)^{\alpha-1}(1-\rho x)^{-\alpha-1} f(\rho x) d \rho .^{2}
\end{aligned}
$$

The transformation

$$
\rho x=1-t^{-1}, \quad x d \rho=t^{-2} d t
$$

yields

$$
\sum_{0}^{\infty} \sigma_{n}^{\alpha} x^{n}=\frac{\alpha}{x} \int_{1}^{1 /(1-x)}\left(t-\frac{t-1}{x}\right)^{\alpha-1} f\left(1-t^{-1}\right) d t .
$$

Putting $x=1-(y+1)^{-1}(y \rightarrow \infty)$ yields

$$
\begin{aligned}
\sum_{0}^{\infty} \sigma_{n}^{\alpha}(1- & \left.\frac{1}{y+1}\right)^{n} \\
& =\alpha\left(1+\frac{1}{y}\right)^{\alpha} \int_{1}^{y+1}\left(1-\frac{t-1}{y}\right)^{\alpha-1} f\left(1-t^{-1}\right) d t .
\end{aligned}
$$

Now set $t=u+1$; then

$$
\begin{aligned}
& \frac{1}{y+1} \sum_{0}^{\infty} \sigma_{n}^{\alpha}\left(1-\frac{1}{y+1}\right)^{n} \\
&=\left(1+\frac{1}{y}\right)^{\alpha-1} \cdot \frac{\alpha}{y} \int_{0}^{\nu}\left(1-\frac{u}{y}\right)^{\alpha-1} \phi(u) d u,
\end{aligned}
$$

where $\phi(u)=f\left(1-(u+1)^{-1}\right)$. Now

$$
\frac{\alpha}{y} \int_{0}^{y}\left(1-\frac{u}{y}\right)^{\alpha-1} \phi(u) d u
$$

is the integral $(C, \alpha)$ transform of the function $\phi(u)$; denoting it by $(\bar{C}, \alpha)$ we have from $(2.4)$

2 The same formula appears in [2, p. 200]. See also E. C. Titchmarsh, The theory of functions, Oxford, 1932, p. 242, example 8. 


$$
A(C, \alpha)\left\{s_{n}\right\}=\left(1+\frac{1}{y}\right)^{\alpha-1}(\bar{C}, \alpha)\{\phi(u)\},
$$

from which our assertion follows, as the right side tends to $s$ for $y \rightarrow \infty$.

3. Abel summability has been generalized to Dirichlet series

$$
D(t)=\sum_{0}^{\infty} a_{n} e^{-\lambda_{n} t}, \quad \text { where } 0 \leqq \lambda_{0}<\lambda_{1}<\cdots, \lambda_{n} \rightarrow \infty .
$$

If the Dirichlet series converges for $t>0$, and if $D(t) \rightarrow s$ as $t \rightarrow 0$, then we write $D_{\lambda} \sum a_{n}=s$. The method is regular. To Abel summability corresponds the case $\lambda_{n}=n, e^{-t}=x$. Furthermore

$$
D(t)=t \sum_{0}^{\infty} s_{n} \int_{\lambda_{n}}^{\lambda_{n+1}} e^{-u t} d u .
$$

Introducing the stepfunction

$$
s(u)= \begin{cases}s_{n}, & \text { for } \lambda_{n} \leqq u<\lambda_{n+1}, n=0,1,2, \cdots, \\ 0, & \text { for } 0 \leqq u<\lambda_{0}, \quad \text { if } \lambda_{0}>0,\end{cases}
$$

we get

$$
D(t)=t \int_{0}^{\infty} s(u) e^{-u t} d u
$$

If $s_{n} \rightarrow s$, then $s(u) \rightarrow s, u \rightarrow \infty$, and $\lim _{t \rightarrow 0} D(t)=s$ defines the generalized limit of $s(u)$.

In general (3.2) is a regular transform of the function $s(u)$, the Laplace transform for which we write $L\{s(u), t\}$. The $(\bar{C}, \alpha)$ means of $s(u)$ are

$$
C_{\alpha}(x) \equiv \alpha x^{-\alpha} \int_{0}^{x}(x-u)^{\alpha-1} s(u) d u, \quad \alpha>0 .
$$

In the case of (3.1) if $\lambda_{n} \leqq x<\lambda_{n+1}$, then $C_{\alpha}(x)$ reduces to

$$
\begin{aligned}
R_{\alpha}(x) & \equiv \alpha x^{-\alpha}\left\{\sum_{0}^{n-1} s_{v} \int_{\lambda_{v}}^{\lambda_{v+1}}(x-u)^{\alpha-1} d u+s_{n} \int_{\lambda_{n}}^{x}(x-u)^{\alpha-1} d u\right\} \\
& =x^{-\alpha} \sum_{0}^{n} a_{v}\left(x-\lambda_{v}\right)^{\alpha} .
\end{aligned}
$$

These are Riesz's typical means of order $\alpha$ (see [1, chap. 4]). If $R_{\alpha}(x) \rightarrow s$, then the series $\sum a_{n}$ is called summable to $s(\lambda, \alpha)$. It is known that $\left[1\right.$, p. 39] if $\sum a_{n}$ is summable $(\lambda, \alpha)$, then it is summable 
$D_{\lambda}$ to the same value. Similarly if $C_{\alpha}(x) \rightarrow s(x \rightarrow \infty)$, then $L\{s(u), t\}$ $\rightarrow s(t \rightarrow 0)$. We have $[1$, p. 39$]$

$$
D(t)=\frac{t^{\alpha+1}}{\Gamma(\alpha+1)} \int_{0}^{\infty} s_{\alpha}(u) e^{-u t} d u,
$$

where $s_{\alpha}(x)=\alpha \int_{0}^{x}(x-u)^{\alpha-1} s(u) d u$. Now

$$
C_{\alpha}(x)=x^{-\alpha} s_{\alpha}(x)
$$

hence

$$
D(t)=\frac{t^{\alpha+1}}{\Gamma(\alpha+1)} \int_{0}^{\infty} u^{\alpha} C_{\alpha}(u) e^{-u t} d u
$$

We have

$$
\Gamma(\alpha)=u^{\alpha} \int_{0}^{\infty} \rho^{\alpha-1} e^{-u \rho} d \rho, \quad \alpha>0,
$$

so that

$$
\begin{aligned}
\left.L\left\{C_{\alpha}(u), t\right)\right\} & =t \int_{0}^{\infty} C_{\alpha}(u) e^{-u t} d u \\
& =\frac{t}{\Gamma(\alpha)} \int_{0}^{\infty} u^{\alpha} C_{\alpha}(u) e^{-u t} \int_{0}^{\infty} \rho^{\alpha-1} e^{-u \rho} d \rho d u \\
& =\frac{t}{\Gamma(\alpha)} \int_{0}^{\infty} \rho^{\alpha-1} \int_{0}^{\infty} s_{\alpha}(u) e^{-u(t+\rho)} d u d \rho \\
& =\alpha t \int_{0}^{\infty} \rho^{\alpha-1}(t+\rho)^{-\alpha-1} D(t+\rho) d \rho .
\end{aligned}
$$

Suppose that $L\{s(u), t\} \rightarrow s$ as $t \rightarrow 0$. Now $\alpha t \int_{0}^{\infty} \rho^{\alpha-1}(t+\rho)^{-\alpha-1} d \rho=1$, hence

$$
L\left\{C_{\alpha}(x), t\right\}-s=\alpha t \int_{0}^{\infty} \rho^{\alpha-1}(t+\rho)^{-\alpha-1}\{D(t+\rho)-s\} d \rho .
$$

But it is easily shown that this expression tends to 0 . Thus the theorem: If

$$
L\{s(u), t\} \rightarrow s \quad \text { as } t \rightarrow 0,
$$

then

$$
L\left\{C_{\alpha}(x), t\right\} \rightarrow s \quad \text { as } t \rightarrow 0
$$


4. We now consider the product of Borel and $(C, \alpha)$ summability. The Borel transform of a sequence $\left\{s_{n}\right\}$ is

$$
B\left\{s_{n}, x\right\}=e^{-x} \sum_{0}^{\infty} \frac{s_{n}}{n !} x^{n} .
$$

We say that $(B) \lim s_{n}=s$, if $\lim _{x \rightarrow \infty} B\left\{s_{n}, x\right\}=s$. The product $B(C, \alpha)$ is the transform

$$
B\left\{\sigma_{n}^{\alpha}, x\right\}=e^{-x} \sum \sigma_{n}^{\alpha} \frac{x^{n}}{n !}
$$

Employing (2.1) and (2.2) we have

$$
\begin{aligned}
\sum_{n=0}^{\infty} \sigma_{n}^{\alpha} \frac{x^{n}}{n !} & =\Gamma(\alpha+1) \sum_{n=0}^{\infty} \frac{x^{n}}{\Gamma(\alpha+n+1)} \sum_{v=0}^{n} s_{v} \gamma_{n-v}^{\alpha-1} \\
& =\frac{\Gamma(\alpha+1)}{\Gamma(\alpha)} \sum_{n=0}^{\infty} \frac{x^{n}}{\Gamma(\alpha+n+1)} \sum_{v=0}^{n} s_{v} \frac{\Gamma(\alpha+n-v)}{(n-v) !} \\
& =\alpha \sum_{v=0}^{\infty} s_{v} \sum_{n=v}^{\infty} x^{n} \frac{\Gamma(\alpha+n-v)}{(n-v) ! \Gamma(\alpha+n+1)}
\end{aligned}
$$

The interchange of summation is legitimate if the double sum is absolutely convergent. From (2.3)

$$
\frac{v ! \Gamma(\alpha+n-v)}{\Gamma(\alpha+n+1)}=\int_{0}^{1} \rho^{\alpha+n-v-1}(1-\rho)^{v} d \rho
$$

hence

$$
\begin{aligned}
\sum_{n=v}^{\infty} x^{n} \frac{\Gamma(\alpha+n-v)}{(n-v) ! \Gamma(\alpha+n+1)} & =\frac{1}{v !} \int_{0}^{1} \rho^{\alpha-1}(1-\rho)^{v} \sum_{n=v}^{\infty} \frac{x^{n}}{(n-v) !} \rho^{n-v} d \rho \\
& =\frac{x^{v}}{v !} \int_{0}^{1} \rho^{\alpha-1}(1-\rho)^{v} e^{\rho x} d \rho .
\end{aligned}
$$

It follows that

$$
\begin{aligned}
\sum_{v=0}^{\infty}\left|s_{v}\right| \sum_{n=v}^{\infty} x^{n} \frac{\Gamma(\alpha+n-v)}{(n-v) ! \Gamma(\alpha+n+1)} & \\
= & \sum_{v=0}^{\infty}\left|s_{v}\right| \frac{x^{v}}{v !} \int_{0}^{1} \rho^{\alpha-1}(1-\rho)^{v} e^{\rho x} d \rho \\
& <\int_{0}^{1} \rho^{\alpha-1} e^{\rho x}\left(\sum_{v=0}^{\infty}\left|s_{v}\right| \frac{x^{v}}{v !}\right) d \rho .
\end{aligned}
$$


By assumption the sum $\sum\left|s_{n}\right| x^{n} / n$ ! is an entire function, hence the double sum on the left is convergent for all $x>0$; this proves that $\sum \sigma_{n}^{\alpha} x^{n} / n$ ! is an entire function, and

$$
\begin{aligned}
\sum_{n=0}^{\infty} \sigma_{n} \frac{x^{n}}{n !} & =\alpha \sum_{v=0}^{\infty} s_{v} \frac{x^{v}}{v !} \int_{0}^{1} \rho^{\alpha-1}(1-\rho)^{v} e^{\rho x} d \rho \\
& =\alpha \int_{0}^{1} \rho^{\alpha-1} e^{\rho x} \sum_{0}^{\infty} s_{v} \frac{x^{v}(1-\rho)^{v}}{v !} d \rho \\
& =\alpha \int_{0}^{1} \rho^{\alpha-1} e^{\rho x} e^{(1-\rho) x} B\left\{s_{n},(1-\rho) x\right\} d \rho
\end{aligned}
$$

Finally

$$
\begin{aligned}
B\left\{\sigma_{n}^{\alpha}, x\right\} & =\alpha \int_{0}^{1} \rho^{\alpha-1} B\left\{s_{n},(1-\rho) x\right\} d \rho \\
& =\frac{\alpha}{x} \int_{0}^{x}\left(1-\frac{t}{x}\right)^{\alpha-1} B\left\{s_{n}, t\right\} d t
\end{aligned}
$$

or

$$
B(C, \alpha)\left\{s_{n}\right\}=(\bar{C}, \alpha) B\left\{s_{n}, t\right\} .
$$

It follows that $(B) \lim s_{n}=s$ implies $(B) \lim \sigma_{n}^{\alpha}=s$. For $\alpha=1$ this result (with a simple proof) was communicated to me by J. Barlaz at the summer meeting, 1949, in Boulder, Colorado.

We finally consider the product of Borel and the generalized Euler transform. The Euler transform $E_{r}$ is defined by

$$
\phi_{n}(r)=\sum_{v=0}^{n} C_{n, v} r^{v}(1-r)^{n-v} s_{v} .
$$

It was shown by K. Knopp that this transform is regular if and only if $r$ is real and $0<r \leqq 1$. Now

$$
\begin{aligned}
B\left\{\phi_{n}(r), x\right\} & =e^{-x} \sum_{0}^{\infty} \phi_{n}(r) \frac{x^{n}}{n !}=e^{-x} \sum_{n=0}^{\infty} \frac{x^{n}}{n !} \sum_{v=0}^{n} C_{n, v} r^{v}(1-r)^{n-v} s_{v} \\
& =e^{-x} \sum_{v=0}^{\infty} s_{v} \frac{r^{v}}{v !}(1-r)^{-v} \sum_{n=v}^{\infty} \frac{x^{n}(1-r)^{n}}{(n-v) !} \\
& =e^{-x} \sum_{0}^{\infty} s_{v} \frac{(r x)^{v}}{v !} e^{(1-r) x}=B\left\{s_{n}, r x\right\} .
\end{aligned}
$$

The interchange of summation is legitimate, the double series being 
absolutely convergent. Hence

$$
B\left\{\phi_{n}(r), x\right\}=B\left\{s_{n}, r x\right\} .
$$

It follows that $(B) \lim s_{n}=s$ implies $(B) \lim \phi_{n}(r)=s$.

\section{REFERENCES}

1. G. H. Hardy and M. Riesz, The general theory of Dirichlet's series, London, 1915.

2. E. Kogbetliantz, Sur la sommation des séries divergentes par les moyennes simples et doubles, Ann. Ecole Norm. (3) vol. 42 (1925) pp. 193-216.

3. Otto Szász, Verallgemeinerung eines Littlewood'schen Satzes über Potenzreihen, J. London Math. Soc. vol. 3 (1928) pp. 254-262.

4. A. Zygmund, Remarque sur la sommabilité des séries de fonctions orthogonales, Bulletin international de l'Academie Polonaise des sciences et des lettres, Classe des Sciences Math. et Nat. Série A (1926) pp. 185-191.

UNIVERSITY OF CincinNaTI

\section{A NOTE ON INDEFINITE INTEGRALS}

\section{J. D. HILL}

I. Throughout the paper $f(x)$ will denote a given function, realvalued and Lebesgue integrable on the interval $X \equiv(0 \leqq x \leqq 1)$. We denote by $E$ the generic measurable subset of $X$ and introduce the following definitions.

(1.1) $F(E)=F(E ; f)=\int_{E} f(x) d x$.

(1.2) $B_{*}(\alpha) \equiv B_{*}(\alpha ; f)$ and $B^{*}(\alpha) \equiv B^{*}(\alpha ; f)$ are, respectively, the greatest lower and least upper bounds of $F(E)$ taken over all sets $E$ of measure $|E|=\alpha(0 \leqq \alpha \leqq 1)$.

Regarded as a function of $E, F(E)$ is called a generalized indefinite integral of $f(x)$ [4] or simply an indefinite integral of $f(x)$ [1]. In this section we develop the principal properties of the functions $B_{*}(\alpha)$ and $B^{*}(\alpha)$, and then obtain as a main result (see (1.9)) the fact that the values of $F(E)$ for $|E|=\alpha$ comprise the closed interval from $B_{*}(\alpha)$ to $B^{*}(\alpha)$. This is an extension of the known fact that $F(E)$ assumes all values between its optimum bounds, where no restrictions are placed on the measures of the sets involved [4]. In the second section the results of the first are applied to the problem of defining a mean value for $F(E)$ as $E$ ranges over the measurable subsets of $X$.

Received by the editors April 26, 1951. 